\title{
Simultaneous Estimation of Sequence and Harmonic Components for StatCom Applications
}

\author{
R.E. Betz ${ }^{1}$, C. Townsend ${ }^{2}$, T.J. Summers ${ }^{3}$, G. Mirzaeva ${ }^{4}$ \\ School of Electrical Engineering and Computer Science \\ University of Newcastle, Australia, 2308 \\ email: ${ }^{1}$ Robert. Betz@newcastle.edu. au \\ ${ }^{2}$ Christopher. Townsend@ newcastle. edu. au \\ ${ }^{3}$ Terry. Summersenewcastle.edu.au \\ ${ }^{4} \mathrm{Galina}$.Mirzaeva@newcastle.edu.au
}

\begin{abstract}
Static Compensators are used to improve power quality. In order to achieve this objective the control system requires accurate knowledge of the positive and negative sequences and user selected harmonics. This paper presents a technique based on an unusual PLL that simultaneously estimates the sequence components and several harmonics. Both simulation and experimental results demonstrating the technique are presented.
\end{abstract}

\section{INTRODUCTION}

In most grid connected power electronic applications accurate estimation of the variables to be controlled is a crucial factor in achieving excellent performance. For example, both the currents and voltages in many power applications have to be accurately estimated in the time domain. This is indeed the case for the Static Compensator (StatCom), which is the power electronic device that is the inspiration for the work in this paper. The measurement system must be robust against noise, and at the same time give reasonable transient performance so that dynamic changes can be handled appropriately.

Another important control issue in power electronic systems is compensating for digital computational delay. Without compensation a digital control system has at least one control period delay between the measurement of the system parameters and associated control computation, and the actual application of the calculated control to the system. If the power electronic switching speed and control frequency is very high this delay may only be small relative to the changes in the grid system parameters over a control interval. However for large VA rated systems the switching speeds cannot be high (so that switching losses can be kept low), and the resultant delay can result in unacceptable phase shifts in the control outputs. These phase shifts become especially important in active filtering applications, since the control would normally be based on the current measurements of the harmonics. The computational delay results in the control being applied in the next control interval cancelling the harmonic value in the previous control interval. By definition the high order harmonic periods are small, and hence the computational delay time manifests itself as a large phase shift in the applied control relative to the period of the harmonic.

One way that computational delay in active filtering can be compensated for is by using accurate predictions of the measurements in the next control interval (i.e. in the future) for the formulation of the control in the current control interval. For this application these predicted measurements are the reference (desired) value to be used in the control calculation. If this can be achieved, then the control is effectively (in steady state at least) being applied with no delay. The waveforms being predicted are in general non-sinusoidal. One technique for achieving very accurate prediction of non-sinusoidal waveforms is to find the trigonometric functions describing the waveform's harmonics, and then use these functions individually to predict forward in time. The estimates are then recombined to form the future prediction of the non-sinusoidal signal. This is the approach that is taken in this paper.

A StatCom control system cannot assume that the grid supply to which it is connected is balanced. Even if there is balance under normal operating conditions, faults in the network, such as single-line-to-ground (SLTG) faults, will result in varying degrees of unbalance, at least until the fault is cleared. The StatCom has to be able to detect the negative sequence and respond in an appropriate manner so that as far as possible it will continue to function correctly. Furthermore, if unbalanced loads are connected to the system and the StatCom is performing a current re-balance control, there is a requirement to be able to accurately measure the load current sequence components to formulate the control references to eliminate the unbalanced currents.

This paper will present a different interconnection structure of PLLs that satisfies the measurement requirements outlined above. The system of PLLs can simultaneously achieve the following:

- estimate the phase and frequency of the supply;

- estimate the positive and negative sequence space 
vectors;

- estimate the space vectors for a user nominated number of harmonics in the supply - both positive and negative sequence harmonics;

- and finally allow an accurate estimate of the time domain values of the signals being processed for one or more control intervals ahead of the current control interval.

\section{THE IDEA}

PLLs have been used for measurement estimation and filtering in power electronic systems for a long time [1][9]. The PLL presented in this paper is different by virtue of the multiple feedback paths available in each PLL that allow multiple PLLs to be connected together in a complex structure to improve performance. To distinguish this structure from others we shall coin the term Advanced PLL (APLL). Figure 1 shows the basic structure of the APLL system when used to estimate only the positive and negative sequence components. If harmonics are also being estimated more APLLs are required, and the already complex feedback is even more complex.

As can be seen from this figure, the positive and negative sequence APLLs have an identical structure except for the Clark transformation at the front of the positive sequence APLL (it is shared by both APLLs). Each APLL has two main components - an amplitude and time domain waveform estimation section, and a phase estimation section. The amplitude and waveform estimation section uses a standard Park Transformation to convert the two phase measurements into a DC component and a double frequency component associated with the opposite sequence, as well as a variety of other frequencies associated with the other harmonics present in the input waveforms. The low pass filter is designed to filter out everything except the synchronous DC $d q$ components. This concept is well established and is a common technique to extract sequence components and harmonics [8], [10]. The vector angle used to implement the Park Transformation is derived from the phase estimation section of the loop. The angle is also applied to the inverse Clark Transformation to reconstruct estimates of the time domain $\alpha \beta$ stationary frame components of the sequence being estimated.

The phase loop operates on the pure phase angle of the particular component of the waveform being estimated. The phase comparator creates direct phase error, and does not rely on the heterodyning principles that are common with traditional analogue PLLs. The phase error generation specifically takes into account that the $\theta$ values in the system are designed to wrap at $\pm \pi$. When the APLL is completely locked $\theta_{\text {err }}$ should be zero by virtue of the PI controller in the feedback loop. The pure integrator that is the equivalent of the traditional voltage controlled oscillator (VCO), as used in analogue PLLs, uses an estimate of the supply frequency as the centre frequency. Notice that the "VCO integrator" is specially implemented so that it wraps the angle at $\pm \pi$. This prevents infinite phase angle $(\theta)$ windup and the inevitable numerical overflow that would occur in a processor, and also ensures that the estimated $\theta_{f p, n}^{\text {est }}$ value is in the valid range for the phase detector to function correctly.

Remark 1: The presence of the PI controller in the estimation loop means that the APLL is able to track the supply with zero frequency and phase error, even under conditions when the supply frequency may not be accurately known. Furthermore, the PI controller allows the APLL to generate an estimate of the true supply frequency.

Most of the features discussed above are not new in the sense that they are present in previously published implementations of PLLs, although not in combination as they are here. However, the novel part of the APLL implementation is the extra feedback path from the two phase time domain outputs to subtract from the input of the other APLLs. For example, $x_{\alpha f p}$ and $x_{\beta f p}$ are subtracted from the $x_{\alpha c}$ and $x_{\beta c}$ composite two phase signals and the remainder is the input to the negative sequence APLL. Clearly if the positive sequence components are being estimated correctly by the positive sequence APLL then this subtraction would only leave the negative sequence as the input to the negative sequence APLL. In a similar fashion the $x_{\alpha f n}$ and $x_{\beta f n}$ signals are subtracted from the composite input to the positive sequence APLL. The approach employed here for the positive and negative sequence components can be generalised for an arbitrary number of APLLs identifying the harmonics as well as the sequence components.

Remark 2: The multiple feedback loops employed in these subtractions is quite complex, and is even more so if the same technique is applied to additional APLLs that are being used for the estimation of the harmonics. Evaluation of the stability and performance boundaries of this approach still has to be done, and will be the subject of a future paper. Having said that, the simulation and experimental studies indicate that the scheme is very robust and stable under typical operating conditions.

\section{Simulation Results}

This section presents results of a number of simulations that were designed to test the feasibility of the basic approach. The simulations are of two types. The first set are implemented in Python, and are signal processing simulations. The waveforms are artificially generated, and then fed through the APLLs and the output can be compared easily with the constituent waveforms that were used to generate the composite input waveform. The second set of simulations are an application of the APLLs to a StatCom based active filter. The filter is modelled as shunt current sources, and the control is implemented digitally. The control period used is twice as long as those used for the 
Positive sequence APLL
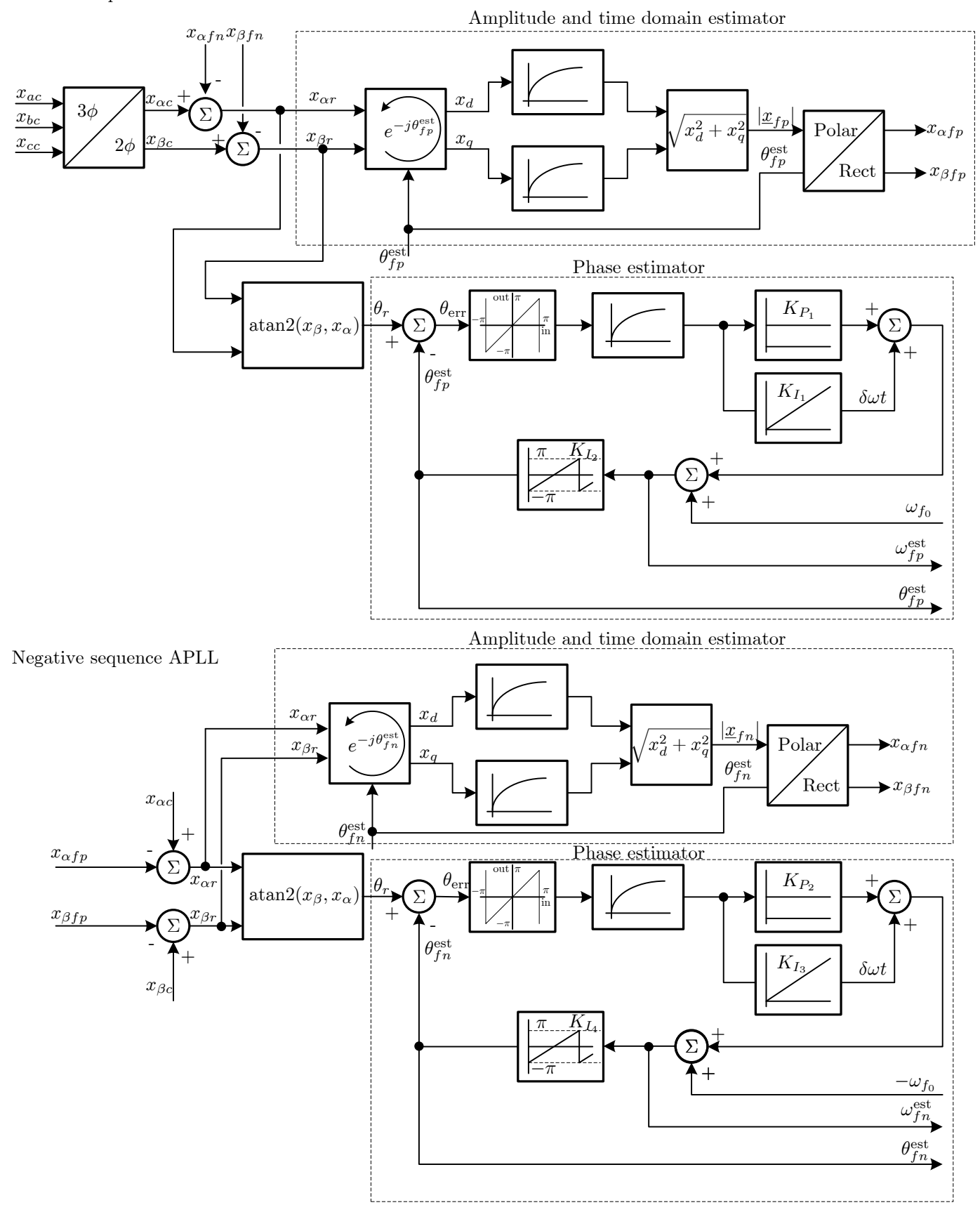

Fig. 1. Block diagram of the structure of an APLL when estimating the positive and negative sequences. 


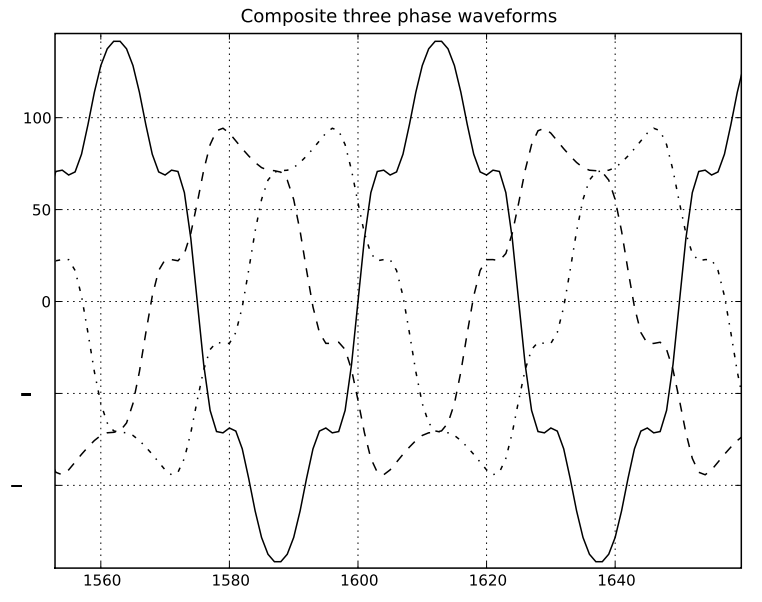

Fig. 2. Composite three phase waveform.

signal processing simulations, so that the effects of control rate can be investigated.

The APLLs were set up in a discrete time Python program to simultaneously extract the positive and negative sequence components, and the 5th and 7th harmonics in both sequences. In order to do this four more APLLs are required in addition to those shown in Figure 1 - two for the positive sequence related 5th and 7th harmonics, and two for the negative sequence related harmonics. Extra feedback paths have to be incorporated into all the loops to account for the extra APLLs. Tables I and II show the amplitudes of the various harmonics for the positive and negative sequences.

\begin{tabular}{|c|c|c|}
\hline 1st & 5th & 7th \\
\hline \hline 100 & 15 & 5 \\
\hline
\end{tabular}

TABLE I

Positive SEQUENCE AMPLITUdes.

\begin{tabular}{|c|c|c|}
\hline 1st & 5th & 7th \\
\hline \hline 30 & 5 & 3 \\
\hline
\end{tabular}

TABLE II

NEgATIVE SEQUENCE AMPLITUDES.

The composite three phase waveform formed from these values is plotted in Figure 2. As can be seen the waveform is very complex, and has a significant amount of unbalance and harmonic pollution. Note that the horizontal axes in all the plots are the sample number, where the sampling rate is $5 \mathrm{kHz}$ (i.e. $200 \mu \mathrm{sec}$ period).

Figure 3 shows the estimated $\alpha$ axis positive sequence waveform. One can see that the true positive sequence and the estimated waveform are so close that they lay on top

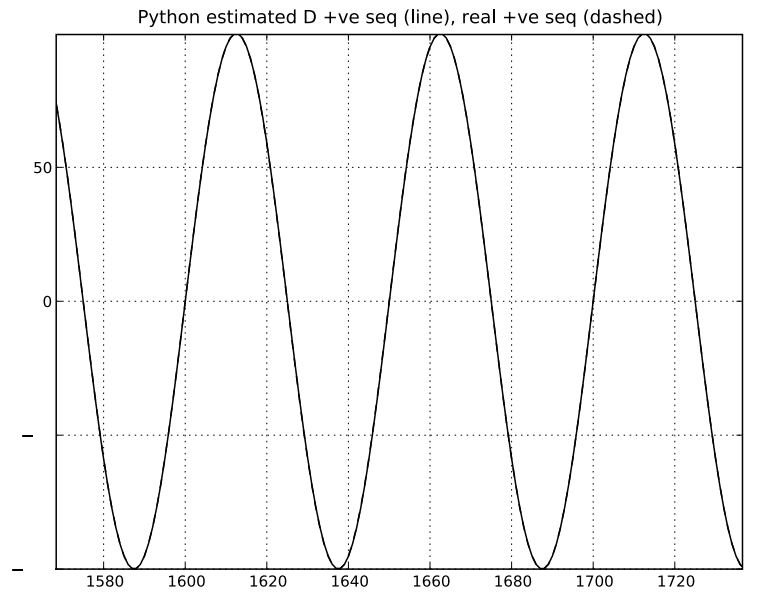

Fig. 3. $\alpha$ axis fundamental waveform for the positive sequence.

of each other. Figure 4 shows that the error between the two waveforms is very small. It also shows the transient error as the APLLs lock onto the waveforms, which takes approximately $300 \mathrm{msec}$. This time is primarily associated with the bandwidth of the APLL control loops, which is suboptimal as it has only been set heuristically in these examples. The design of the loop bandwidths will be a main focus of the theoretical analysis to be presented in a future paper.

Remark 3: The simulation code produces the estimate one time step ahead of the current time step. These estimates are stored in an array where the index into the array aligns with the real data values. This allows the future estimates to be directly compared with the corresponding true signal sample. This is the reason that there is no relative delay in the waveforms.

The only apriori knowledge used in the APLLs is an estimate of the supply frequency and related harmonics. If these estimates have significant error the performance of the system is not compromised as the phase loop quickly identifies the frequency error and compensates. In steady state the phase errors achieved in the APLLs is of the order of milli-radians.

Figures 5 and 6 show the same results for the negative sequence waveforms.

It should be noted that when APLLs have been used for the identification of harmonics it has been found that they are very robust with respect to noise and glitches in the supply, and still provide excellent estimates. Furthermore, it has also been shown that the APLL technique for harmonic extraction is superior to other PLL techniques in terms of transient and steady state performance [11], [12]. These same properties carry over to the sequence estimation application.

Finally let us consider the harmonics. We shall only show 


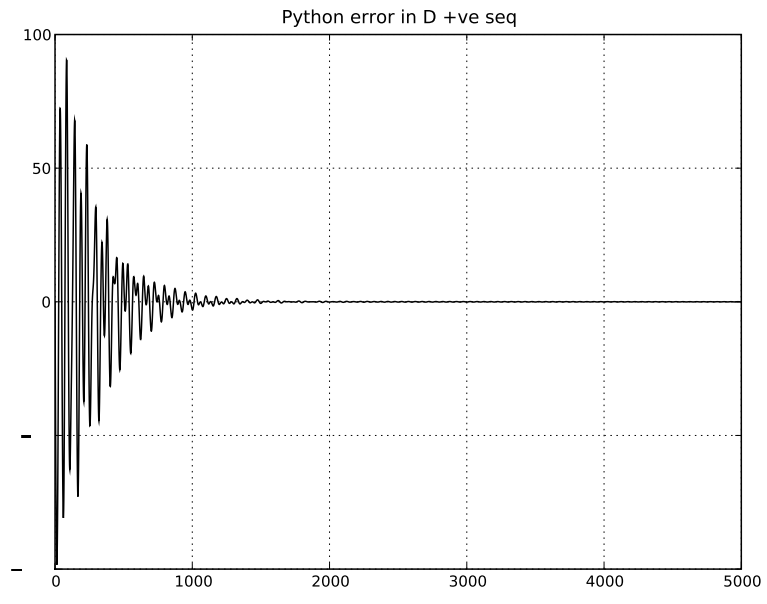

Fig. 4. $\alpha$ axis fundamental waveform positive sequence error.

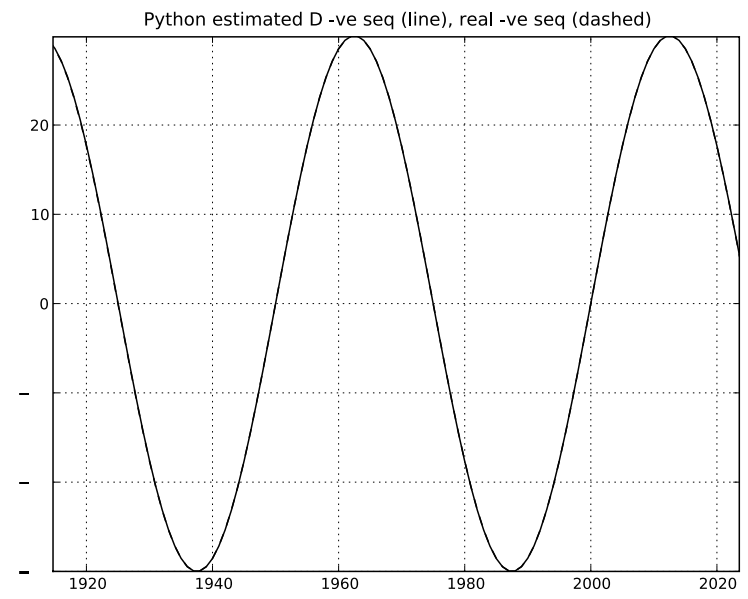

Fig. 5. $\alpha$ axis fundamental waveform for the negative sequence.

the plots for the $\alpha$ axis 5th and 7th harmonics for the negative sequence, as these are also representative of the performance of the positive sequence harmonic estimation. There are two plots in these figures - the estimate and the actual harmonic. In both cases they are superimposed on each other. The waveforms do not appear sinusoidal because of the interaction of the sampling time and the frequency of the harmonic waveforms.

Remark 4: Given that the amplitude and frequency of the sinusoidal waveforms are accurately predicted by the APLLs it is possible to accurate predict forward at least one control interval.

The other simulation study implemented was an active filter. The simulation was implemented in the Saber ${ }^{\circledR}$ simulation package, and consisted of a simple three phase power network with a voltage source, line impedances, and

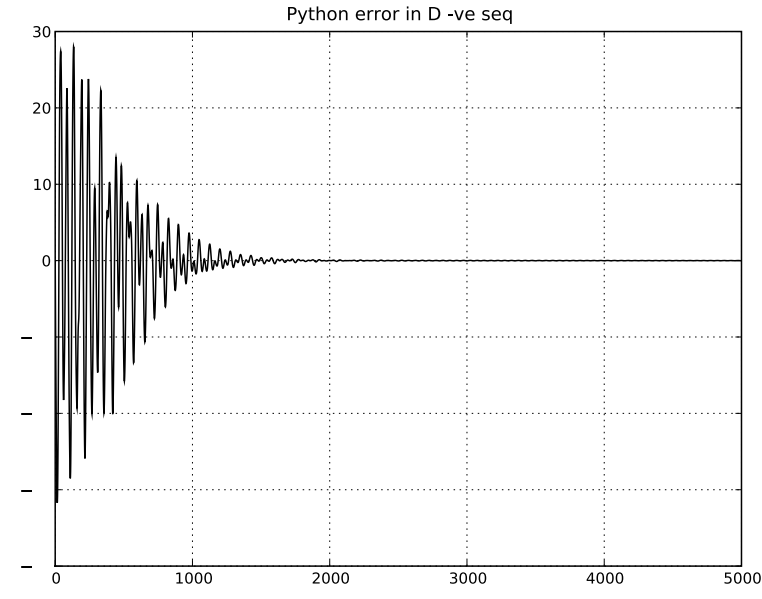

Fig. 6. $\quad \alpha$ axis fundamental waveform negative sequence error.

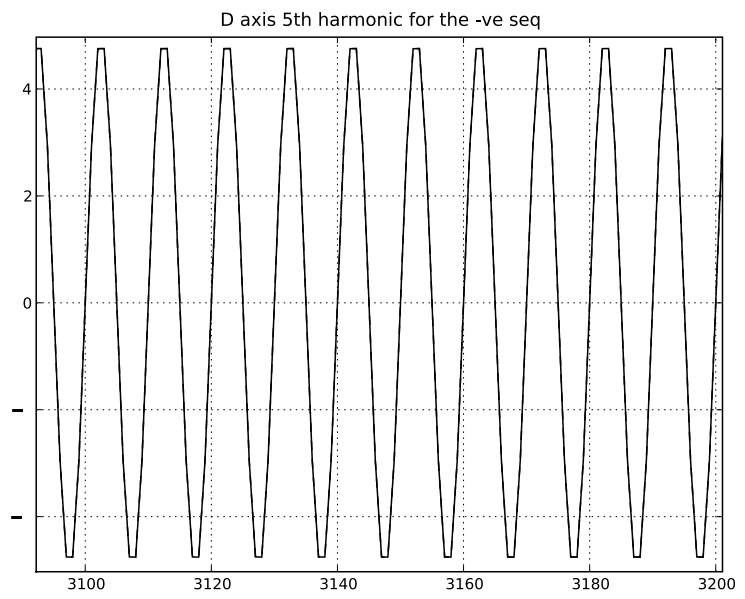

Fig. 7. $\alpha$ axis negative sequence 5th harmonic waveforms.

a non-linear load consisting of an array of AC current sinks at the fundamental and harmonic frequencies to simulate an unbalanced harmonic load. The use of the current sinks allows one to precisely know the harmonic content and degree of unbalance in the load current waveforms. The active filter section was implemented as a idealised current source based StatCom - i.e. the currents were injected into the power network using shunt ideal current sources. The APLLs in the Saber ${ }^{\circledR}$ were implemented as 'C' dynamic linked libraries (DLLs) so that the same code could be used for the experimental system.

Figure 9 shows a block diagram of this simulation. Note the complex configuration of the APLLs for this case with 5 inputs feeding back into each of the APLLs from the other APLLs. In this simulation the active filter currents consist of the all of the estimated current components except for the 


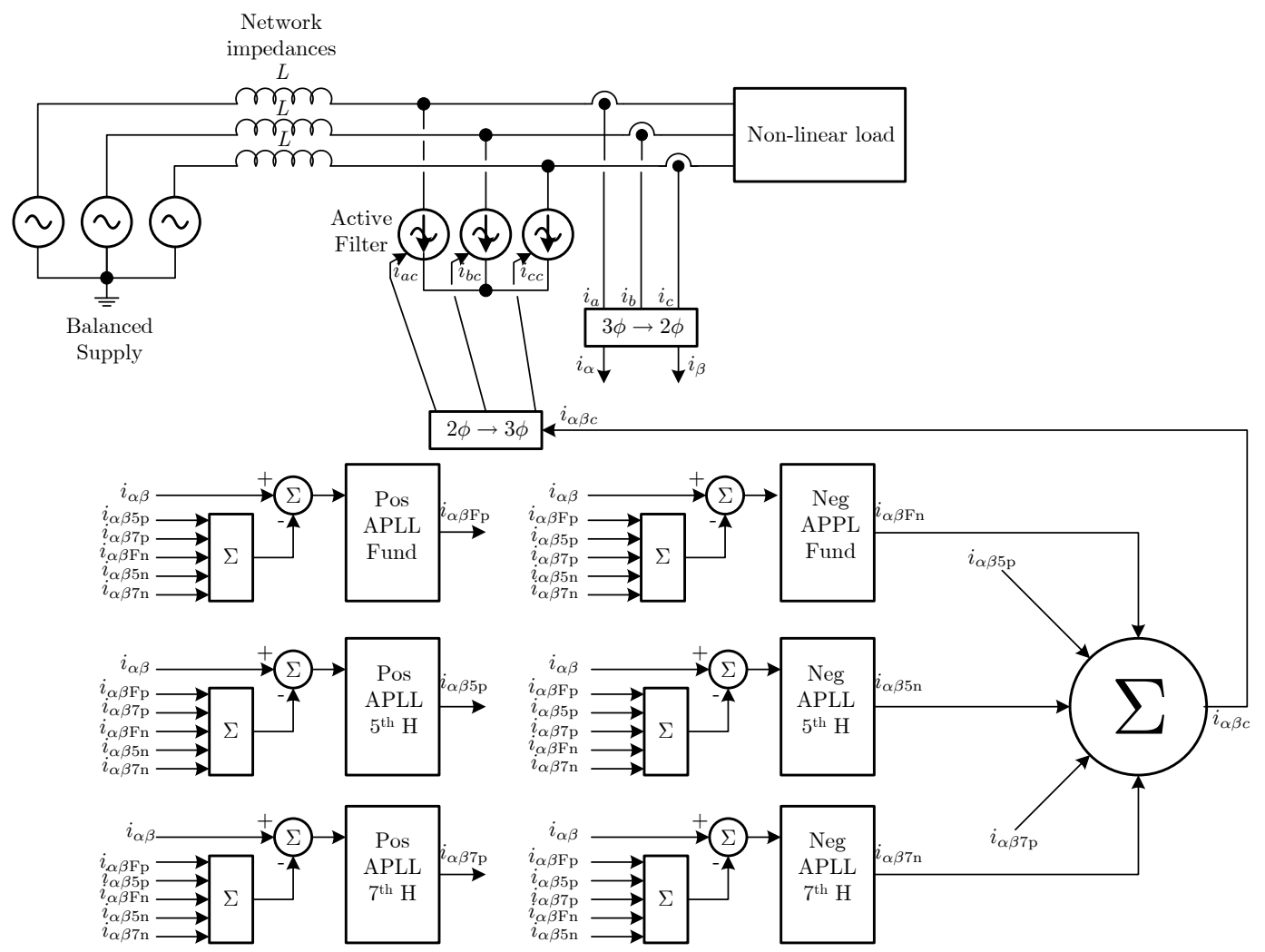

Fig. 9. Block diagram of active filter simulation.

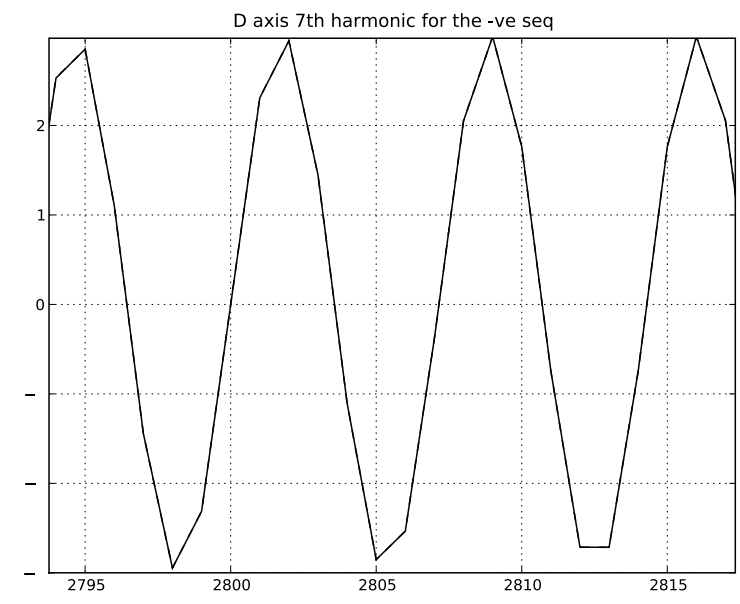

Fig. 8. $\alpha$ axis negative sequence 5th harmonic waveforms.

positive sequence current. In other words the active filter is supplying the harmonic currents and the negative sequence required by the load. Therefore the current flowing from the source should only be the positive sequence current.

Remark 5: The fact that the current flowing through the network inductances when the active filter is active is si- nusoidal means that the point-of-common-coupling voltage will also be sinusoidal.

In order to test the performance of the APLLs in this application a sampling period of $400 \mu \mathrm{sec}$ was chosen as this corresponds to the control rate used in the multilevel StatCom that the authors have constructed, and also also corresponds to the rate used for the experiments. The current sources in the load have been set to have the harmonic amplitudes as shown in Tables I and II. The overall performance of the system can be seen in Figure 10. As can be seen from this figure, the active filter is turned on at time 0.5 secs. Prior to this the current flowing from the supply is unbalanced and has a high harmonic content. When the filter is turned on the currents drawn from the supply become balanced and the harmonics are removed. One can see that the ' $a$ ' phase active filter current being injected to compensate for the harmonics and the unbalance is highly non-linear. The performance indicates that the APLLs are doing an excellent job of identifying the sequence components and their related harmonics one control period ahead.

Figures 11 and 12 show the positive and negative sequence, actual and estimated fundamental and 5th harmonics (the 7th is similar). As can be seen the APLLs accurately predict the values of the components. 


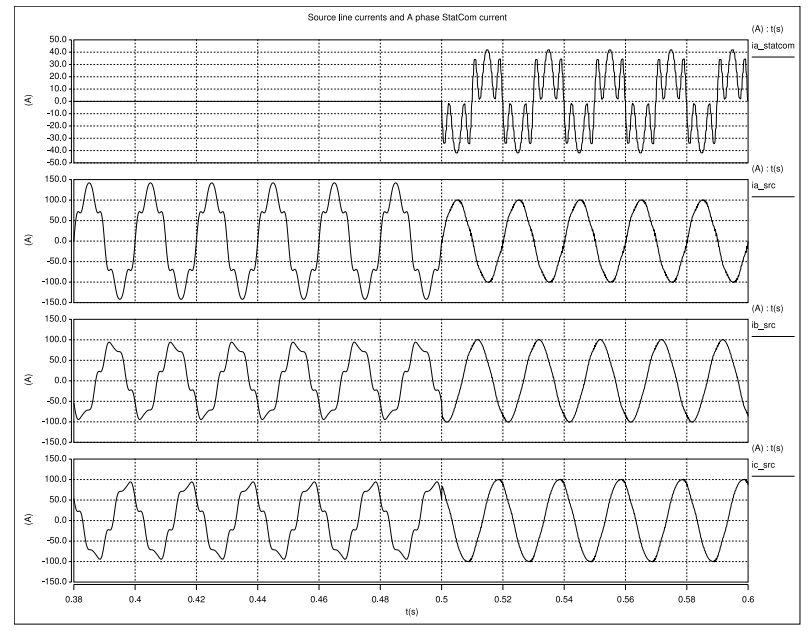

Fig. 10. Plot of the performance of the Active Filter using APLL estimators.

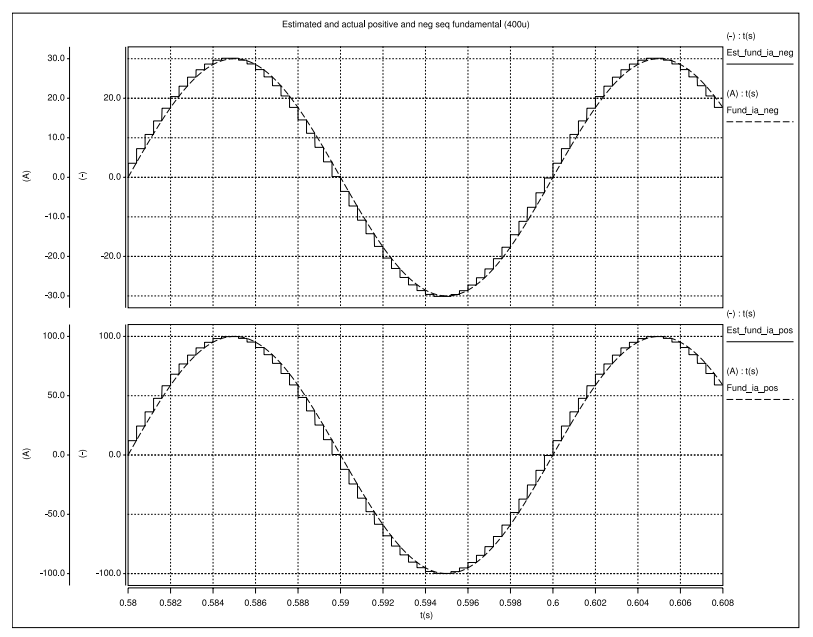

Fig. 11. Simulation showing fundamental estimation.

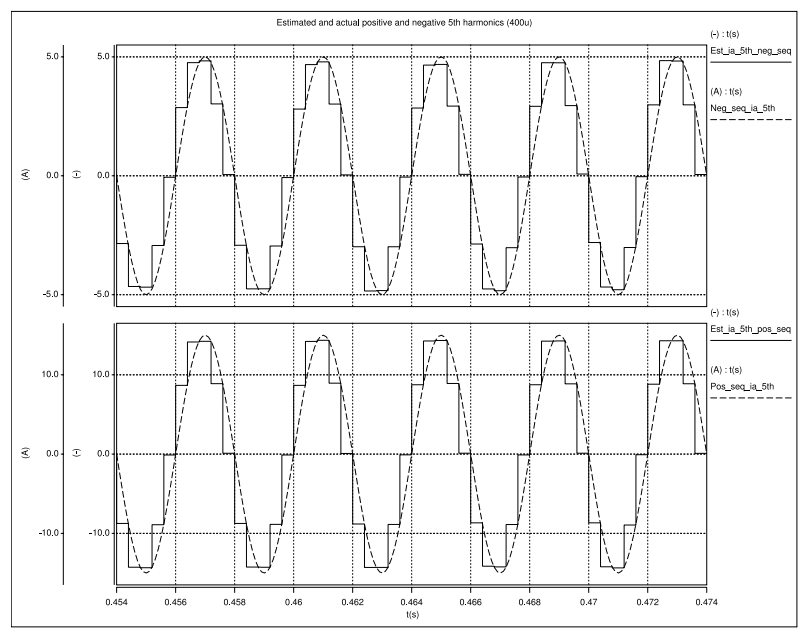

Fig. 12. Simulation showing 5th harmonic estimation.

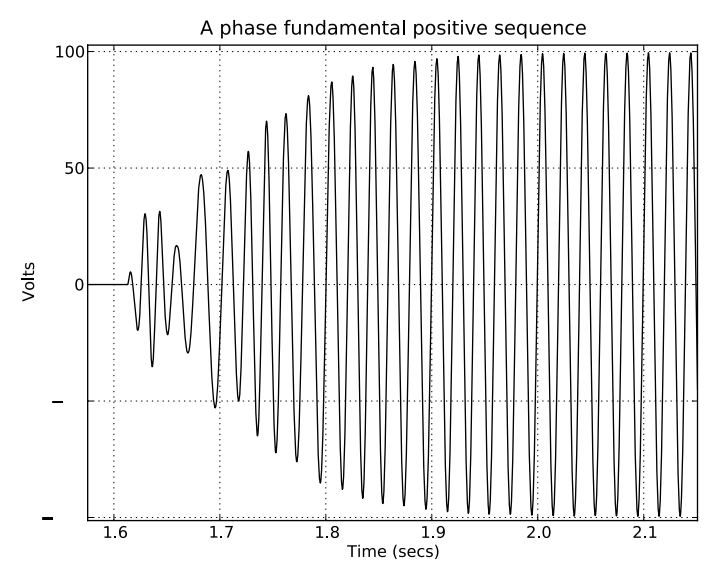

Fig. 13. Experimental estimates of the positive sequence fundamental.

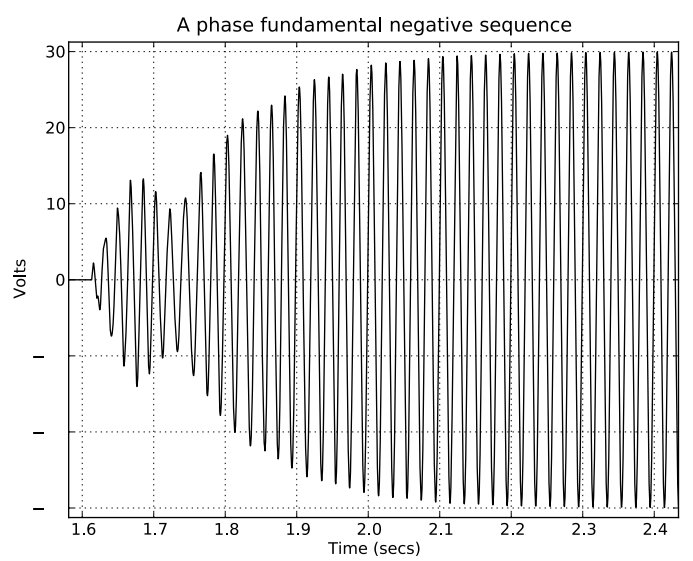

Fig. 14. Experimental estimates of the negative sequence fundamental

\section{EXPERIMENTAL RESUlts}

In order to further verify the performance of the APLLs the DLLs used in the simulation were modified slightly and embedded into the real-time environment used as part of an existing multilevel StatCom system [13], [14]. This system also has a programmable power supply that allows unbalance as well as harmonics to be programmed into the supply voltages. The control system hardware sampled these voltages with a sampling period of $400 \mu$ secs and then implemented in software a similar APLL structure to that shown in Figure 9. This was used to identify the sequence and harmonic components, and the results were output to files to be plotted.

Figures 13 and 14 show the identified positive and negative fundamental sequence components from the experimental data. These plots also show the start-up transient, and indicate that it takes approximately $300 \mathrm{msec}$ for the APLL output to converge to the correct value from initial values of zero for the outputs. 


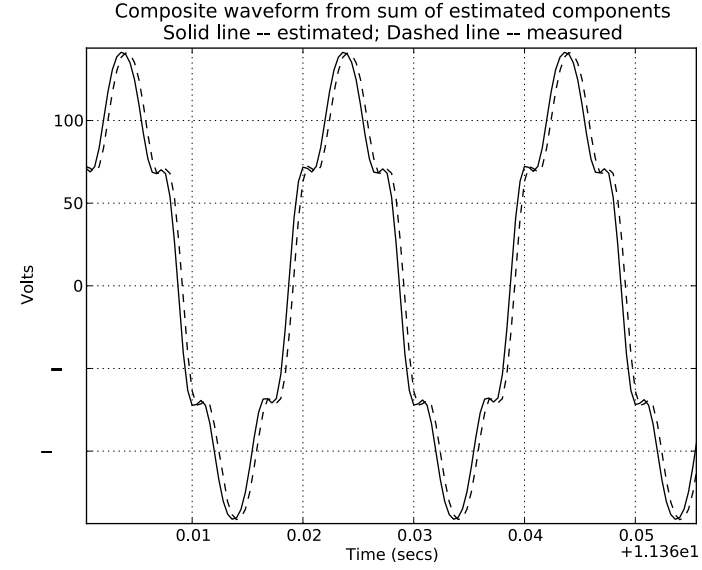

Fig. 15. Composite waveform formed from adding together the experimental estimates of the components.

It was difficult to determine the accuracy of the sequence and harmonic components produced using the same technique used in the simulations. This was due to the fact that the individual components were not available as separate waveforms from the power supply. Instead an indirect technique was used. If the components from the APLLs are correct, then when they are all added together the resultant waveform should be the same as the waveform from the power supply. Figure 15 is the result of carrying out the summation of the experimentally obtained component waveforms. As can be seen, the composite waveform follows closely the actual waveform, indicating that the estimated components are very accurate. The leading phase shift of the estimated composite waveform is a consequence of the fact that the estimates are one sample period ahead of the actual waveform, as required to eliminate digital delay.

\section{CONClusions}

This paper has outlined a different way of connecting together a group of phase locked loops in order to simultaneously separate out the sequence and harmonic components of waveforms typical in power systems and power electronic applications. The technique also eliminates the digital delay typically encountered in discrete time implementations. The individual components of the PLLs are well known - it is the way they are connected together with the multiple feedback loops that is different. This approach has been shown previously to give superior performance for the extraction of harmonics and sequence components as compared to other PLL approaches [11], [12]. The use of the APLLs to simultaneouly separate out the positive and negative sequence components with harmonics present is novel and challenging.

The paper presented two simulations of the technique. The first is a signal processing based simulation, where the algorithms are implemented digitally and are used to process synthetically generated data that contains a high degree of unbalance, and significant harmonics superimposed on both the positive and negative sequences. The second simulation is application based, where a dynamic simulation of an active filter is developed, and the APLLs are used to derive the references for the active filter. The input waveforms to the APLLs have the same constituent components as the signal processing simulation. In both simulations the performance of the APLLs is excellent.

As a final verification, the APLL technique was implemented in a real-time environment on the hardware used for a multilevel StatCom. As with the simulations the estimates of the sequence and harmonic components is excellent. The paper has demonstrated that the technique is a robust technique for sequence and harmonic extraction.

\section{REFERENCES}

[1] H. Akagi, E. H. Watanabe, and M. Aredes, Instantaneous Power Theory and Application to Power Conditioning. John Wiley and Sons, 2007.

[2] M. Karimi-Ghartemani and M. R. Iravani, "A new phase-locked loop (PLL) system," in Circuits and Systems, 2001. MWSCAS 2001. Proceedings of the 44th IEEE 2001 Midwest Symposium on, vol. 1, (Dayton, OH), pp. 421-424, Aug. 2001.

[3] M. Karimi-Ghartemani and M. R. Iravani, "A nonlinear adaptive filter for online signal analysis in power systems: applications," IEEE Trans. Power Del., vol. 17, pp. 617-622, Apr. 2002.

[4] M. Karimi-Ghartemani and M. Iravani, "A method for synchronization of power electronic converters in polluted and variablefrequency environments," Power Systems, IEEE Transactions on, vol. 19, pp. 1263-1270, Aug. 2004.

[5] M. Karimi-Ghartemani and M. R. Iravani, "Measurement of harmonics/inter-harmonics of time-varying frequencies," IEEE Trans. Power Del., vol. 20, pp. 23-31, Jan. 2005.

[6] M. Karimi-Ghartemani, "A novel three-phase magnitude-phaselocked loop system," Circuits and Systems I: Regular Papers, IEEE Transactions on [see also Circuits and Systems I: Fundamental Theory and Applications, IEEE Transactions on], vol. 53, pp. 17921802, Aug. 2006.

[7] M. Karimi-Ghartmani, M. Iravani, and F. Katiraei, "Extraction of signals for harmonics, reactive current and network-unbalance compensation," IEE Proceedings - Generation, Transmission and Distribution, vol. 152, pp. 137-143, January 2005.

[8] C. Hochgraf and R. H. Lasseter, "Statcom controls for operation with unbalanced voltages," IEEE Transactions on Power Delivery, vol. 13, pp. 538-544, Apr. 1998.

[9] N. I. A. Wahab, N. Mariun, A. Mohamed, and M. Mohamad, "Response of d-STATCOM under unbalanced voltage condition caused by SLG fault," in Research and Development, 2003. SCORED 2003. Proceedings. Student Conference on, pp. 395-400, Aug. 2003.

[10] H. Akagi, S. Inoue, and T. Yoshii, "Control and performance of a transformerless cascade PWM STATCOM with star configuration," IEEE Trans. Ind. Appl., vol. 43, pp. 1041-1049, July/Aug. 2007.

[11] R. Betz, T. Summers, and G. Mirzaeva, "Comparison of three harmonic extraction techniques for active filters," in Proceedings of the Australasian Universities Power Engineering Conference (AUPEC2009), September 2009.

[12] R. Betz, T. Summers, and G. Mirzaeva, "Active filtering and var control of a cascaded h-bridge multi-level statcom," November 2009.

[13] R. E. Betz, T. Summers, and B. Cook., "Outline of the control design for a cascaded h-bridge statcom," in Proceedings of the IEEE-IAS Annual Meeting, Edmonton Canada, Oct 2008. ISBN 978-1-4244$2279-1$.

[14] R. Betz and T. Summers, "Design and development of an $11 \mathrm{kv} \mathrm{H}-$ Bridge multilevel STATCOM," in Australasian Universities Power Engineering Conference - AUPEC 2007, (Perth, Australia), Engineers Australia, December 2007. ISBN: 978-0-646-47499-1. 